\title{
Welcome to the International Journal of Indigenous Health (IJIH)
}

Globally, disparities in Indigenous health status demonstrate inequity across a number of indicators. Social determinants, including systemic marginalization and discrimination, contribute to create high-risk environments for Indigenous peoples. Moreover, lack of health services as well as barriers to access contribute to an unacceptable gap in life expectancy, mortality and wellness between Indigenous peoples and other populations within the same country. Although it is essential to examine persistent health disparities, it has been increasingly acknowledged that exclusively examining poor health status can serve to pathologize individuals, communities and populations, particularly within colonial contexts. As well, this deficit model ignores the strength, resistance and innovation of Indigenous peoples, which deserves exploration and promotion. A growing cadre of Indigenous health researchers, committed to ethical and mutually beneficial partnerships with communities, are utilizing Indigenous research and knowledge translation approaches to transform and re-define this diverse and exciting multidisciplinary field of study.

The International Journal of Indigenous Health (IJIH) is pleased to welcome new and long-time readers of the Journal of Aboriginal Health (JAH). The original JAH has been renamed, re-branded and expanded to international authors with the objective to increase sharing of knowledge across borders, nations, communities and cultures. In 2006, the National Aboriginal Health Organization (NAHO) created the JAH with the aspiration to make health research more accessible to Aboriginal communities. With an expanding international readership, this practice continued until NAHO closed its doors in 2012. At NAHO's request, the Aboriginal Health Research Networks Secretariat (AHRNetS), hosted by the Centre for Aboriginal Health Research, University of Victoria, is honoured to continue this important work and is now publishing the journal under its new name. AHRNetS is the national coordinating body of the Network Environments for Aboriginal Health Research (NEAHR), a national capacity-building initiative of the Canadian Institutes for Health Research - Institute of Aboriginal People's Health.

The IJIH is a space for sharing transformative approaches to Indigenous health research and interventions. In keeping with ongoing international developments, the IJIH has adopted the internationally supported principle of self-definition for Indigenous communities. The term Indigenous is therefore used as an umbrella term for self-defined and diverse Indigenous nations, communities, languages and culture groups.

In this inaugural IJIH edition, we are pleased to share a collection of excellent papers, which span diverse countries, cultures, disciplines and critical health issues, with a particular focus on Indigenous ways of knowing, decolonizing methodologies, suicide prevention, food security, child malnutrition, health research ethics reform, and sexual health communication with youth. Fern Brunger and her colleagues report on a consultative process in Newfoundland and Labrador, Canada that resulted in key changes to the governance of Indigenous health research ethics review in that province. Sarah Flicker and her colleagues explore the efficacy of strengthbased, art-making workshops as a decolonizing research method designed to improve health promotion among diverse Indigenous youth groups across Canada. Richard Hovey and his colleagues discuss Indigenous ways of knowing and their connection to diabetes prevention in a Kanien'kehá:ka community in Quebec, Canada. Michael Kral and his colleagues examine the 
issue of Inuit youth suicide in Arctic Canada and its connection to the impact of colonization on interpersonal relationships with a view to informing suicide prevention strategies. Jenny Munro and her colleagues conduct an intersectionality analysis of gender, Indigeneity and food insecurity among ultra-poor, Indigenous Garo women in Bangladesh with the aim of increasing attention on poverty alleviation. Elizabeth Rink and her colleagues delve into Inuit parent/guardian-youth sexual health communication in Greenland and identify potential changes to the design of community based sexual health education programs. Heather Wehr and her colleagues review gender and household roles in Indigenous Maya communities in Guatemala to guide future nutritional interventions aimed at preventing chronic child malnutrition.

On behalf of our volunteer peer reviewers, Editorial Advisory Board members, submitting authors, Interns, volunteers and staff, we hope that you find this, our very first issue of the IJIH, both informative and inspiring. We also hope you will become involved with IJIH in the future, by sharing your knowledge and expertise as well as innovative approaches to research and community health. In the spirit of working together for the improved health of current and future generations of Indigenous peoples, we thank you for your ongoing support and welcome new readers to the IJIH.

In Solidarity,



Charlotte Loppie - Editor

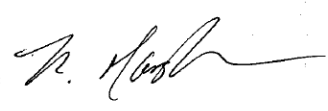

Namaste Marsden - Managing Editor 